\title{
Genetic background contributions to the disproportion of vitamin A deficiency in pregnant women of certain ethnicities in populations of the United States.
}

\author{
Tao Wang \\ Albert Einstein College of Medicine \\ Diana J Garretto \\ Stony Brook University Hospital \\ Carmen R. Isasi \\ Albert Einstein College of Medicine \\ Wellington V Cardoso \\ Columbia University \\ John M. Greally \\ Albert Einstein College of Medicine \\ Loredana Quadro \\ Rutgers University
}

Masako Suzuki ( $\nabla$ masako.suzuki@einsteinmed.org)

Albert Einstein College of Medicine https://orcid.org/0000-0003-0605-9225

Short report

Keywords: Vitamin A deficiency, pregnant females, Hispanics, NHANES

Posted Date: August 2nd, 2020

DOI: https://doi.org/10.21203/rs.3.rs-49188/v1

License: (c) (i) This work is licensed under a Creative Commons Attribution 4.0 International License.

Read Full License 


\section{Abstract}

Vitamin A is an essential micronutrient that plays critical roles in many biological functions of the body. Limited access to vitamin A-rich food or supplements severely affects tissue and blood levels of vitamin A. Therefore, low serum vitamin A and poverty levels are strongly associated in vitamin A deficiency (VAD) studies that have focused mainly on developing countries. The current national prevalence rate of vitamin A deficiency in the United States is reported to be very low $(<1 \%)$. However, several studies, including ours, have suggested that people from certain ethnic groups still face a higher proportion of vitamin $A$ deficiency. Here, we re-analyzed two different datasets of serum retinol levels of pregnant females to assess the VAD status differences between women of different ancestries. We found that pregnant females with non-Hispanic Black and with Latin American/Afro-Caribbean ancestry have strikingly high proportions of vitamin A deficiency. Moreover, we identified candidate genetic variants that associate with the disproportions between these different ancestries. Maternal vitamin A deficiency increases the risk of adverse health outcomes for both the mother and offspring later in life. Measuring serum retinol levels of pregnant women in the higher risk groups and provision of food interventions based on genetic information to improve the vitamin A status of at-risk women are needed. Our study strongly suggests that emergency actions need to be taken to reduce vitamin A deficiency in specific, at-risk ethnic groups.

\section{Main Text}

The essential micronutrient vitamin A plays critical roles in vision $(1,2)$, immune system $(3,4)$, cell growth and differentiation (5-8), as well as in the development of multiple organs, including the lung, heart, eyes, and kidneys (reviewed in $(9,10)$ ). Since vitamin A is an essential micronutrient, limited access to vitamin Arich food or supplements severely affects tissue and blood levels of vitamin $A$ in humans (11). In the United States (U.S.), while the current prevalence rate of vitamin A deficiency (VAD) - defined as serum retinol levels lower than $1.05 \mu \mathrm{mol} / \mathrm{l}$ - is estimated to be less than 1\% (CDC's Second Nutrition Report (12)), it varies between different ethnic groups and races in the U.S. (13). By using datasets from the National Health and Nutrition Examination Survey (NHANES) that measured vitamin A (retinol and its esterified form, retinyl ester) and provitamin A carotenoids in the serum of over 24,000 individuals in 2001-2002, 2003-2004, and 2005-2006 (14), we found that the sample-weighted serum retinol levels of vitamin A ranged from 1.74 (non-Hispanic Black) to $2.09 \mu \mathrm{mol} / \mathrm{L}$ (non-Hispanic White) (Additional file 1). For this analysis we employed sample weights as recommended by the $\mathrm{CDC}$ to produce an unbiased national estimates (14). Moreover, an epidemiological study based on the NHANES datasets demonstrated that non-Hispanic Black and Mexican American females of childbearing age have a higher rate of VAD (15). However, since the overall prevalence of VAD is considered to be rare in developed countries, vitamin A levels have not been measured routinely and populations levels of the vitamin are not up to date (16). Therefore, the sparse available data have failed to detect the up-to-date status of VAD for individuals in developed countries.

Recently, we reported a high VAD proportion rate among pregnant females in the Bronx, NY, USA (17), where the ethnic diversity and the poverty rate is much higher than in the rest of the nation (12). The 
original study addressed the effects of bariatric surgery on serum vitamin A levels during pregnancy (17). The most surprising result we observed in the study was that more than $60 \%$ of the pregnant women who did not undergo the bariatric surgery (control group) had serum retinol levels lower than $1.05 \mu \mathrm{mol} / \mathrm{l}(17)$, i.e., they were vitamin A deficient. The proportion of vitamin A deficient females in the Bronx is much higher than that of non-Hispanic Black and Hispanic (Mexican American) females of the same age group reported by Hanson et al. (15). This finding prompted us to test the disproportionality of VAD status in pregnant females between different ethnic groups by re-analyzing our data from the Brox study (17) as well as those from NHANES (18). While the NHANES data were collected almost two decades ago, this is the latest and most current nationwide serum vitamin A level assessment in the United States.

We evaluated serum retinol levels, the measure of VAD status (11,19-21), identifying distinct ancestries of participants in two separate datasets, and tested the possibility that genetic variation could contribute to these disproportionalities using three publicly available allele frequency databases. As the first serum retinol level dataset, we re-analyzed the third trimester serum retinol levels in the Bronx dataset by ethnic group, specifically using the self-reported ethnicities (non-Hispanic Black, Hispanic, or other race) as the ethnicities (17). As the second dataset, we analyzed the pregnant serum retinol levels by ethnicities using the reported race/ethnicity (RIDRETH1) information in the Sample Person Demographics Files from the NHANES dataset (14). To identify the genetic variants which might be associated with the differences in the proportions of VAD between ethnic groups, we tested the deviations of allele frequency between the groups. The allele frequency of the variants of each ethnic group were obtained from Allele Frequency Aggregator (ALFA) (22), the Population Architecture using Genomics and Epidemiology (PAGE (23), BioProject Accession: PRJNA168052), and 1000 Genomes project (24). The associations of genetic variants with gene expression levels, defining expression quantitative trait loci (eQTL), were obtained from the Genotype-Tissue Expression project, GTEx (25).

From the Bronx study, we only used the third-trimester serum retinol levels were measured for 67 women out of the 96 participants (17). We found no significant association between missing data status and other covariates. While maternal serum levels of beta-carotene, the most abundant dietary vitamin $\mathrm{A}$ precursor (11), and cord blood serum retinol were found to be significantly associated with maternal VAD status ( $p=0.041$ and $p=0.007$, respectively), other known covariates, including the bariatric surgery status, did not show significant associations (Table 1). The proportion of VAD in Hispanic women was $65.9 \%$ (29 out of 44 Hispanic participants), in non-Hispanic Blacks was 53.3\% (8 of 15 African American participants), and for other ethnicities was $37.5 \%$ (3 in 8 participants). Among Hispanic participants ( $\mathrm{n}=$ 44), vitamin A deficient women tended to be younger than the vitamin A sufficient women $(p=0.088)$, but education levels, pre-pregnancy body mass index (BMI), and gestational weight gain (GWG) were not associated with the VAD status $(p=0.876, p=0.195$, and $p=0.935$, respectively). Unfortunately, poverty levels of each cohort was not assessed in the Bronx study (17). However, in the Bronx, degree of education and poverty level are generally negatively correlated (poverty rate of less than High School is $38.97 \%$, High School is $24.45 \%$, and College is $18.87 \%$ ) (26). Therefore, the poverty levels of vitamin A deficient and sufficient females might be similar in the Bronx study, and we assume that the poverty level might not be directly associated with VAD status between ethnicity. Also, no clinical evidence was available for these 
VAD pregnant females as they had not been assessed since their deficiency should be considered subclinical based on serum levels. Despite the relatively limited sample size of the Bronx study, we found that the proportion of VAD in Hispanic women was higher in African American women and both the proportions were much higher than the estimated levels in the U.S. (Additional file 1). In the NHANES dataset the proportion of VAD among Hispanic (Mexican American) pregnant females was half that of non-Hispanic Black pregnant females, $14.9 \%$ and $32.0 \%$ respectively $(p<0.0001$, Fisher's exact test, Additional file 1; 966 pregnant females). From this latter dataset, we focused our analysis on pregnant women aged 17-42 (similar to the age range of the Bronx study) with a poverty income ratio less than 1.85 (27). Although the proportions of VAD were slightly increased in both ethnic groups, the proportions of VAD among non-Hispanic Black pregnant women remained more than two times higher than that of Hispanic (Mexican American) pregnant women (15.9\% in Hispanic (Mexican American) and $36.3 \%$ in nonHispanic Black, 447 pregnant females). This suggests that the VAD status difference between these two minority groups might be independent from their poverty levels.

The major origins of Hispanic populations in the Bronx are Latin Americans with Afro-Caribbean ancestry (39.4\% Dominicans and 36.4\% Puerto Ricans, 2010 U.S. Census data (26)), whereas the Hispanic population in the NHANES data we used is Mexican Americans. Thus, we asked whether the ancestryspecific genetic variations might contribute to lower serum retinol levels independent from the poverty levels that reflect the nutritional intakes (27). Genetic contributions to the levels of circulating retinol have been reported in European populations. Specifically, a family study in France showed that the heritability estimate for serum retinol concentration (30.5\%) was much larger than the variability accounted for by household, i.e., individuals living in the same house (14.2\%) (28). Moreover, in the GWAS Catalog (29), two single nucleotide polymorphisms (SNPs) (rs10882272 T/C and rs1667255 C/A), identified from a genomewide association study of 5,006 "Caucasian" males, are listed as associated with serum retinol levels (30). The association of rs 10882272 was replicated in independent samples, including 3,792 females and 504 males (30). In this same study, differences in the strength of the SNP associations between males and females were also reported. However, the rate of VAD in the individuals studied was low, thus the authors were not able to test the association of the genetic variation with VAD (30). Unfortunately, genotypic information of the cohorts we used, the Bronx study and the NHANES datasets, is not available; therefore, we compared the low serum retinol allele frequencies of rs10882272 and rs 1667255 between different ethnic groups in publicly available datasets, ALFA and PAGE, to assess if this genetic variation could be associated with VAD status differences between racial/different ethnic groups. While we did not observe significant differences in major allele frequencies of rs 1667255 between Hispanic groups (Additional file 2), as we predicted, the allele frequencies of rs 10882272 showed significant variation between different ethnic groups. The frequency of the allele associated with low serum retinol was much higher in African (0.620) and African American (0.617) compared to European (0.383) and Asian (0.106) individuals in the ALFA dataset (Fig. 1a). Similarly, the PAGE dataset results showed that the risk allele frequencies were higher in Latin Americans with Afro-Caribbean ancestry [Puerto Ricans (0.455), Dominicans (0.502) and Cubans (0.410)] compared to Mexicans (0.260), Central Americans (0.288), South Americans (0.278) or Native Americans (0.357) (Fig. 1b). The ethnic groups with the higher VAD proportions showed a higher frequency of the allele associated with low serum retinol levels. The rs 10882272 variant is located in the $3^{\prime}$ 
UTR of the free fatty acid receptor 4 (FFAR4) gene and downstream of the retinol binding protein 4 (RBP4) gene. The FFAR4 gene encodes a GPCR receptor (GPCR120) for free long-chain fatty acids, including omega-3 $(31,32)$. FFAR4/GPCR120 is expressed in various cell types, including pituitary, lung, macrophages, adipocytes, intestinal neuroendocrine cells and pancreatic cells. Thus, it participates in a number of physiological processes, including energy regulation, insulin sensitivity, immunological homeostasis and anti-inflammatory responses (33). RBP4 is the sole specific carrier for retinol in the bloodstream $(2,34)$. Predominantly expressed in the hepatocytes, RBP4 binds retinol to mobilize vitamin A from the liver, the primary body storage site of the vitamin, towards the peripheral tissues (2). We tested if the rs 10882272 is an expression quantitative trait locus (eQTL) for its nearby genes using a publicly available database (the Genotype-Tissue Expression project, GTEx) (25). In the GTEx data, we detected the associations between the rs 10882272 variations and the expression levels of RBP4 in the liver where the gene is highly expressed (35), with the presence of the allele associated with low serum retinol levels also associated with increased expression of RBP4 (normalized effect size: $0.137, \mathrm{p}=0.00012$, and $\mathrm{m}$-value 0.987). For FFAR4, while we detected the association in lung (normalized effect size: $0.126, p=8.5 e-6$, and $\mathrm{m}$-value 1.00), but not in pituitary (normalized effect size: $0.0334, \mathrm{p}=0.5$, and $\mathrm{m}$-value 0.809 ). The pituitary showed highest expression of FFAR4 in the GTEx data.

Another genetic variant associated with differences in serum retinol levels between different ethnic groups is the rs738409 polymorphism (36), which is located in the patatin-like phospholipase domain containing 3 (PNPLA3) gene. PNPLA3 encodes a gene involved in the mobilization of retinyl esters stored in stellate cells $(36,37)$. The rs 738409 polymorphism is a missense variant, the $\mathrm{C}$ to $\mathrm{G}$ nucleotide substitution changing the amino acid I[ATC] to M[ATG]. The PNPLA3 I148M missense variant is a loss-of-function mutation (36), and the associations between the variant and the risk of nonalcoholic fatty liver disease (NAFLD) has been reported (38-40). The frequency of the mutant allele varies between ethnic groups (0.144 to 0.499 , African American to Mexican; PAGE dataset). Of note, individuals homozygous for PNPLA3 I148M have lower circulating levels of RBP4 (36). Changes in circulating levels of RBP4 have been linked to pathological conditions and variations in nutritional intake (41-44). Interestingly, reported associations of the circulating levels of RBP4 and NAFLD are conflicting, and a recent meta analysis reported that circulating RBP4 levels may indeed not be associated with NAFLD (45). Thus, we speculate that the associations between PNPLA3 I148M variants and circulating RBP4 levels might be independent of NAFLD status. In animal models, while retinol deficiency leads to accumulation of RBP4 in liver, likely by inhibiting its hepatic secretion, the RBP4 mRNA levels in the liver show no differences between vitamin A deficiency and sufficiency (46). Further studies are needed to test the associations of these SNPs with circulating RBP4 levels, serum retinol levels, and disease status.

Not only these three SNPs, but also several GWAS and candidate gene association studies have also identified other polymorphisms associated with serum retinol and beta-carotene levels and the betacarotene bioactivities (47). We thus also assessed the allele frequency deviations of the 39 associated with circulating vitamin A levels (47) between different ethnic groups in the 1000 Genome Project (24). The deviations of allele frequencies of those vitamin A related SNPs between different ethnic groups are listed in the Additional file 2. The average of the allele frequency standard deviation among ethnic groups 
was 0.122 , significantly higher than randomly selected sets of 39 SNPs from the 1000 Genomes data $(p=$ 0.030 , permutation test with 1,000 iterations, Additional file 1). Since serum retinol level variations between different ethnic groups have been reported, this result is not surprising. However, this is the first systematic analysis of the allele frequency variations of the vitamin A-related SNPs among different ethnic groups. Looker et al. reported serum retinol level differences among three Hispanic groups using the Hispanic Health and Nutrition Examination Survey (HHANES) conducted from 1982-1984 (48). The authors found that Mexican Americans have a higher VAD prevalence rate than Puerto Ricans or Cubans in both adults and children. This study was performed almost four decades ago when the participants' nutrient status might have differed from the current one. The CDC's Second Nutrition Report in 2012 showed that serum vitamin A concentrations increased between 1999-2000 and 2005-2006 in the United States (geometric mean, $52.8 \mu \mathrm{g} / \mathrm{dl}$ to $54.7 \mu \mathrm{g} / \mathrm{dl}$ ). Moreover, serum retinyl palmitate levels, an indicator of newly ingested vitamin $A$, were dramatically increased during this period in Mexican Americans (geometric mean $0.759 \mathrm{ug} / \mathrm{dl}$ in 1999-2000 to $1.85 \mathrm{ug} / \mathrm{dl}$ in 2005-2006) (49). The differences observed in 19821984 might reflect the lower dietary intake of vitamin A at that time and not genetic variation. An updated population-based measurement of serum retinol levels is clearly needed.

We acknowledge that there are several limitations to our study: the limited sample size of the Bronx cohort $(n=97)$, the poverty levels nor clinical information on VAD-related outcome of the Bronx cohort was not availale, the serum retinol data of NHANES were collected more than a decade ago, and the genotype information of all participants is not available in both cohorts. Further genome-wide association studies with demographic information, including food accessibility/intake in multiethnic cohorts, are needed to assess the influences of genetic variation and the different VAD status between different ethnic groups.

In summary, while VAD in developed countries is believed to be a rare condition, there is a substantial proportion of VAD pregnant females of certain ethnic groups, even in wealthy, developed countries. While the WHO does not recommend routine vitamin A supplementation to pregnant women, but they recommend vitamin A supplementation to pregnant women in a given geographical area if $\geq 20 \%$ of pregnant women have serum retinol levels $<0.70 \mu \mathrm{mol} / \mathrm{L}(50)$. Our re-analysis of the Bronx study showed that more than $40 \%$ of pregnant women have serum retinol $<0.70 \mu \mathrm{mol} / \mathrm{L}$, strongly suggesting that urgent actions need to be taken to reduce the VAD, especially in unusually susceptible ethnic groups to reduce the risk of adverse health conditions of the mother (51) and diseases of offspring later in life $(52,53)$. Moreover, our results showed that genetic variations may be contributing to the VAD status differences between ethnic groups, at least in pregnant women. Further understanding of this association will ultimately enable adequate food interventions based on the genetic information could be crucial to improve maternal vitamin A status during pregnancy in these higher risk groups.

\section{Abbreviations}

eQTL

expression quantitative trait loci

GWAS 
Genome-Wide Association Study

NHANES

National Health and Nutrition Examination Survey

PAGE

Population Architecture using Genomics and Epidemiology

VAD

vitamin A deficiency

\section{Declarations}

\section{Availability of data and materials:}

The serum retinol and demographic information of the Bronx dataset, originally published in the J Perinat Med (PMID:30231012), are available by request. The National Health and Nutrition Examination Survey (NHANES) datasets were downloaded from the NHANES repository (https://www.cdc.gov/nchs/nhanes/index.htm) and merged file in accordance with the NHANES guidelines and recommendations.

\section{Funding:}

This work was supported by the National Heart, Lung, and Blood Institute of the National Institutes of Health under award number 1R01HL145302. The content is solely the responsibility of the authors and does not necessarily represent the official views of the National Institutes of Health.

\section{Author information:}

\section{Affiliations}

Department of Genetics, Albert Einstein College of Medicine, Bronx, NY, USA

John M. Greally and Masako Suzuki

Department of Epidemiology and Population Health, Albert Einstein College of Medicine, Bronx, NY, USA

Tao Wang and Carmen R. Isasi

Department of Obstetrics and Gynecology and Women's Health, Stony Brook University Medical Center, Stony Brook, NY, USA

Diana Garretto

Department of Medicine, Columbia University, New York, NY USA

Wellington V. Cardoso 
Department of Food Science and Rutgers Center for Lipid Research, and New Jersey Institute for Food, Nutrition, and Health, Rutgers University, New Brunswick, NJ, USA

Loredana Quadro

\section{Contributions}

Wrote manuscript draft (MS,LQ), prepared illustrations (MS), approved final manuscript (MS, DG, TW, CRI, WC, JMG, LQ), conceived project (MS, LQ), analyzed data (MS), formulated research questions (MS, LQ), interpreted results (MS, TW, DG, CRI, JMG, WC, LQ), led investigation (MS). All authors read and approved the final manuscript.

\section{Corresponding author}

Correspondence to Masako Suzuki

\section{Ethics declarations:}

\section{Ethics approval and consent to participate}

We used publicly available data that no ethical approval is required.

\section{Consent for publication}

Not applicable.

\section{Competing interests}

All authors declare no competing interest.

\section{References}

1. Vogel S, Piantedosi R, O’Byrne SM, Kako Y, Quadro L, Gottesman ME, et al. Retinol-binding proteindeficient mice: biochemical basis for impaired vision. Biochemistry. 2002 Dec 24;41(51):15360-8.

2. Quadro L, Blaner WS, Salchow DJ, Vogel S, Piantedosi R, Gouras P, et al. Impaired retinal function and vitamin A availability in mice lacking retinol-binding protein. EMBO J. 1999 Sep 1;18(17):4633-44.

3. Liu X, Cui T, Li Y, Wang Y, Wang Q, Li X, et al. Vitamin A supplementation in early life enhances the intestinal immune response of rats with gestational vitamin A deficiency by increasing the number of immune cells. PLoS One. 2014 Dec 11;9(12):e114934.

4. Sirisinha S. The pleiotropic role of vitamin A in regulating mucosal immunity. Asian Pac J Allergy Immunol. 2015 Jun;33(2):71-89.

5. Williams JB, Napoli JL. Metabolism of retinoic acid and retinol during differentiation of F9 embryonal carcinoma cells. Proc Natl Acad Sci USA. 1985 Jul;82(14):4658-62. 
6. Lee TF, Mak KM, Rackovsky O, Lin Y-L, Kwong AJ, Loke JC, et al. Downregulation of hepatic stellate cell activation by retinol and palmitate mediated by adipose differentiation-related protein (ADRP). J Cell Physiol. 2010 Jun;223(3):648-57.

7. Cammas L, Romand R, Fraulob V, Mura C, Dollé P. Expression of the murine retinol dehydrogenase 10 (Rdh10) gene correlates with many sites of retinoid signalling during embryogenesis and organ differentiation. Dev Dyn. 2007 Oct;236(10):2899-908.

8. Janick-Buckner D, Barua AB, Olson JA. Induction of HL-60 cell differentiation by water-soluble and nitrogen-containing conjugates of retinoic acid and retinol. FASEB J. 1991 Mar 1;5(3):320-5.

9. Marx J, Naudé H, Pretorius E. The Effects of Hypo- and Hypervitaminosis a and Its Involvement in Foetal Nervous System Development and Post-Natal Sensorimotor Functioning - A Review. The British Journal of Development Disabilities. 2006 Jan;52(102):47-64.

10. Zile MH. Vitamin A and embryonic development: an overview. J Nutr. 1998 Feb;128(2 Suppl):455S458 .

11. Tanumihardjo SA, Russell RM, Stephensen CB, Gannon BM, Craft NE, Haskell MJ, et al. Biomarkers of Nutrition for Development (BOND)-Vitamin A Review. J Nutr. 2016 Aug 10;146(9):1816S-48S.

12. https://www.cdc.gov/nutritionreport/pdf/4page_\%202nd\%20nutrition\%20report_508_032912.pdf [Internet]. [cited 2020 May 22]. Available from:

https://www.cdc.gov/nutritionreport/pdf/4page_\%202nd\%20nutrition\%20report_508_032912.pdf

13. Hanson MA, Gluckman PD. Developmental origins of health and disease: new insights. Basic Clin Pharmacol Toxicol. 2008 Feb;102(2):90-3.

14. National Health and Nutrition Examination Surveys (NHANES) [Internet]. Centers for Disease Control and Prevention (CDC). National Center for Health Statistics (NCHS). National Health and Nutrition Examination Survey Data. Hyattsville, MD: U.S. Department of Health and Human Services, Centers for Disease Control and Prevention. [cited 2020 Jul 13]. Available from:

https://wwwn.cdc.gov/nchs/nhanes/Default.aspx

15. Hanson C, Lyden E, Abresch C, Anderson-Berry A. Serum retinol concentrations, race, and socioeconomic status in of women of childbearing age in the united states. Nutrients. 2016 Aug $19 ; 8(8)$.

16. Wirth JP, Petry N, Tanumihardjo SA, Rogers LM, McLean E, Greig A, et al. Vitamin A Supplementation Programs and Country-Level Evidence of Vitamin A Deficiency. Nutrients. 2017 Feb 24;9(3).

17. Garretto D, Kim Y-K, Quadro L, Rhodas RR, Pimentel V, Crnosija NA, et al. Vitamin A and $\beta$-carotene in pregnant and breastfeeding post-bariatric women in an urban population. J Perinat Med. 2019 Feb 25;47(2):183-9.

18. The National Health and Nutrition Examination Survey (NHANES).

19. de Pee S, Dary O. Biochemical indicators of vitamin A deficiency: serum retinol and serum retinol binding protein. J Nutr. 2002;132(9 Suppl):2895S-2901S.

20. World Health Organization. Indicators for Assessing Vitamin A Deficiency and their Application in Monitoring and Evaluating Intervention Programmes [Internet]. 1996 [cited 2020 Jul 13]. Available 
from:

https://www.who.int/nutrition/publications/micronutrients/vitamin_a_deficiency/WHONUT96.10.pdf? ua $=1$

21. Palmer AC, West KP, Dalmiya N, Schultink W. The use and interpretation of serum retinol distributions in evaluating the public health impact of vitamin A programmes. Public Health Nutr. 2012 Jul;15(7):1201-15.

22. Allele Frequency Aggregator [Internet]. [cited 2020 Jul 13]. Available from: https://www.ncbi.nlm.nih.gov/snp/docs/gsr/alfa/

23. Population Architecture using Genomics andEpidemiology [Internet]. [cited 2020 Jul 13]. Available from: https://www.pagestudy.org/index.php/home

24. 1000 Genomes Project Consortium, Abecasis GR, Auton A, Brooks LD, DePristo MA, Durbin RM, et al. An integrated map of genetic variation from 1,092 human genomes. Nature. 2012 Nov $1 ; 491(7422): 56-65$.

25. GTEx Consortium. Human genomics. The Genotype-Tissue Expression (GTEx) pilot analysis: multitissue gene regulation in humans. Science. 2015 May 8;348(6235):648-60.

26. U.S. Census Bureau. US Census [Internet]. [cited 2020 Jul 13]. Available from: https://data.census.gov/cedsci/

27. Blumberg JB, Frei B, Fulgoni VL, Weaver CM, Zeisel SH. Contribution of dietary supplements to nutritional adequacy by socioeconomic subgroups in adults of the united states. Nutrients. 2017 Dec $22 ; 10(1)$.

28. Gueguen S, Leroy P, Gueguen R, Siest G, Visvikis S, Herbeth B. Genetic and environmental contributions to serum retinol and alpha-tocopherol concentrations: the Stanislas Family Study. Am J Clin Nutr. 2005 May;81(5):1034-44.

29. The NHGRI-EBI Catalog of human genome-wide association studies. GWAS Catalog [Internet]. [cited 2020 Jul 13]. Available from: https://www.ebi.ac.uk/gwas/

30. Mondul AM, Yu K, Wheeler W, Zhang H, Weinstein SJ, Major JM, et al. Genome-wide association study of circulating retinol levels. Hum Mol Genet. 2011 Dec 1;20(23):4724-31.

31. Oh DY, Talukdar S, Bae EJ, Imamura T, Morinaga H, Fan W, et al. GPR120 is an omega-3 fatty acid receptor mediating potent anti-inflammatory and insulin-sensitizing effects. Cell. 2010 Sep 3;142(5):687-98.

32. Hirasawa A, Tsumaya K, Awaji T, Katsuma S, Adachi T, Yamada M, et al. Free fatty acids regulate gut incretin glucagon-like peptide-1 secretion through GPR120. Nat Med. 2005 Jan;11(1):90-4.

33. Oh DY, Walenta E. Omega-3 Fatty Acids and FFAR4. Front Endocrinol (Lausanne). 2014 Jul 16;5:115.

34. Quadro L, Hamberger L, Colantuoni V, Gottesman ME, Blaner WS. Understanding the physiological role of retinol-binding protein in vitamin A metabolism using transgenic and knockout mouse models. Mol Aspects Med. 2003 Dec;24(6):421-30.

35. Thompson SJ, Sargsyan A, Lee S-A, Yuen JJ, Cai J, Smalling R, et al. Hepatocytes are the principal source of circulating RBP4 in mice. Diabetes. 2017 Jan;66(1):58-63. 
36. Pirazzi C, Valenti L, Motta BM, Pingitore P, Hedfalk K, Mancina RM, et al. PNPLA3 has retinyl-palmitate lipase activity in human hepatic stellate cells. Hum Mol Genet. 2014 Aug 1;23(15):4077-85.

37. Kovarova M, Königsrainer I, Königsrainer A, Machicao F, Häring H-U, Schleicher E, et al. The Genetic Variant I148M in PNPLA3 Is Associated With Increased Hepatic Retinyl-Palmitate Storage in Humans. J Clin Endocrinol Metab. 2015 Dec;100(12):E1568-74.

38. Tian C, Stokowski RP, Kershenobich D, Ballinger DG, Hinds DA. Variant in PNPLA3 is associated with alcoholic liver disease. Nat Genet. 2010 Jan;42(1):21-3.

39. He S, McPhaul C, Li JZ, Garuti R, Kinch L, Grishin NV, et al. A sequence variation (I148M) in PNPLA3 associated with nonalcoholic fatty liver disease disrupts triglyceride hydrolysis. J Biol Chem. 2010 Feb 26;285(9):6706-15.

40. Romeo S, Kozlitina J, Xing C, Pertsemlidis A, Cox D, Pennacchio LA, et al. Genetic variation in PNPLA3 confers susceptibility to nonalcoholic fatty liver disease. Nat Genet. 2008 Dec;40(12):1461-5.

41. Graham TE, Yang Q, Blüher M, Hammarstedt A, Ciaraldi TP, Henry RR, et al. Retinol-binding protein 4 and insulin resistance in lean, obese, and diabetic subjects. N Engl J Med. $2006 \mathrm{Jun}$ 15;354(24):2552-63.

42. Manolescu D-C, Sima A, Bhat PV. All-trans retinoic acid lowers serum retinol-binding protein 4 concentrations and increases insulin sensitivity in diabetic mice. J Nutr. 2010 Feb;140(2):311-6.

43. Aeberli I, Biebinger R, Lehmann R, L'allemand D, Spinas GA, Zimmermann MB. Serum retinol-binding protein 4 concentration and its ratio to serum retinol are associated with obesity and metabolic syndrome components in children. J Clin Endocrinol Metab. 2007 Nov;92(11):4359-65.

44. Jing L, Xiao M, Dong H, Lin J, Chen G, Ling W, et al. Serum Carotenoids Are Inversely Associated with RBP4 and Other Inflammatory Markers in Middle-Aged and Elderly Adults. Nutrients. 2018 Feb $25 ; 10(3)$.

45. Zhou Z, Chen H, Ju H, Sun M. Circulating retinol binding protein 4 levels in nonalcoholic fatty liver disease: a systematic review and meta-analysis. Lipids Health Dis. 2017 Sep 20;16(1):180.

46. Blaner WS. Retinol-binding protein: the serum transport protein for vitamin A. Endocr Rev. 1989 Aug;10(3):308-16.

47. Borel P, Desmarchelier C. Genetic Variations Associated with Vitamin A Status and Vitamin A Bioavailability. Nutrients. 2017 Mar 8;9(3).

48. Looker AC, Johnson CL, Underwood BA. Serum retinol levels of persons aged 4-74 years from three Hispanic groups. Am J Clin Nutr. 1988 Dec;48(6):1490-6.

49. Centers for Disease Control and Prevention. Second Nutrition Report (2012) [Internet]. [cited 2020 Jul 13]. Available from: https://www.cdc.gov/nutritionreport/report_2012.html

50. Vitamin A supplementation during pregnancy [Internet]. [cited 2020 Jul 13]. Available from: https://www.who.int/elena/titles/guidance_summaries/vitamina_pregnancy/en/

51. Radhika MS, Bhaskaram P, Balakrishna N, Ramalakshmi BA, Devi S, Kumar BS. Effects of vitamin A deficiency during pregnancy on maternal and child health. BJOG. 2002 Jun;109(6):689-93. 
52. Bhat PV, Manolescu D-C. Role of vitamin A in determining nephron mass and possible relationship to hypertension. J Nutr. 2008 Aug;138(8):1407-10.

53. Chen F, Marquez H, Kim Y-K, Qian J, Shao F, Fine A, et al. Prenatal retinoid deficiency leads to airway hyperresponsiveness in adult mice. J Clin Invest. 2014 Feb;124(2):801-11.

\section{Table 1}

Due to technical limitations Table 1 is available as a download in the Supplementary Files.

\section{Figures}

a ALFA

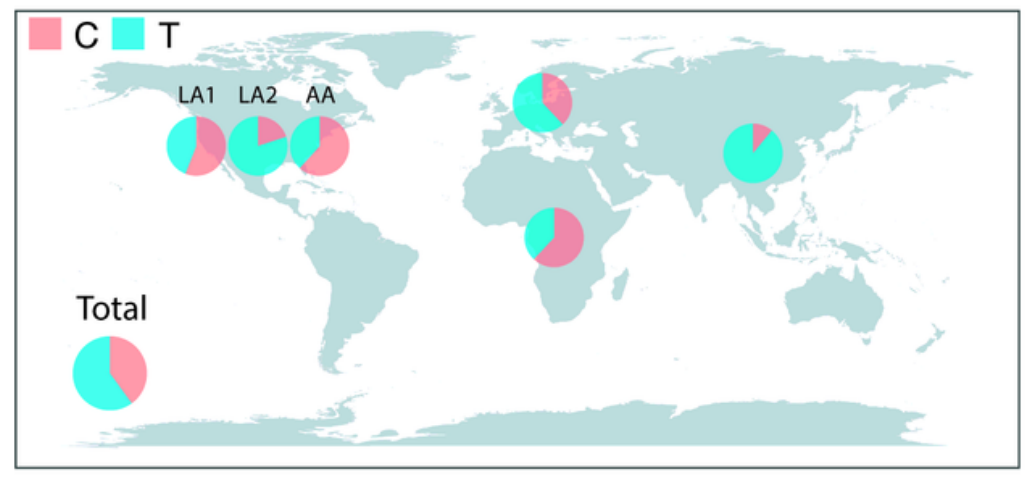

b PAGE rs 10882272

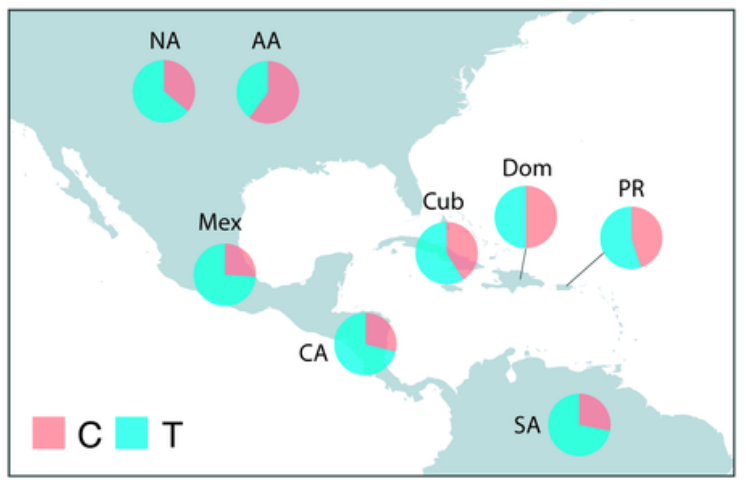

Figure 1

\section{Figure 1}

Variations of the allele frequencies of rs 10882272 We plotted the allele frequency of each ethnic group (a) Allele Frequency Aggregator (ALFA) and (b) Population Architecture using Genomics and Epidemiology (PAGE). LA1, Latin American 1 (represented by Puerto Rican and Dominican); LA2, Latin American 2 (represented by Mexican and Mexican American); AA, African American; NA, Native American; CA, Central American; SA, South American; Cub, Cuban; Dom, Dominican; Mex, Mexican; PR, Puerto Rican . Note: The designations employed and the presentation of the material on this map do not imply the expression of any opinion whatsoever on the part of Research Square concerning the legal status of any country, territory, city or area or of its authorities, or concerning the delimitation of its frontiers or boundaries. This map has been provided by the authors.

\section{Supplementary Files}

This is a list of supplementary files associated with this preprint. Click to download.

- AdditionalFile1.pdf 
- Additionalfile2.xls

- Table1.xls 